\title{
Generalized Ablowitz-Ladik hierarchy in topological string theory
}

\author{
Kanehisa Takasaki * \\ Graduate School of Human and Environmental Studies \\ Kyoto University \\ Sakyo, Kyoto 606-8501, Japan
}

\begin{abstract}
This paper addresses the issue of integrable structures in topological string theory on generalized conifolds. Open string amplitudes of this theory can be expressed as the matrix elements of an operator on the Fock space of $2 \mathrm{D}$ charged free fermion fields. The generating function of these amplitudes with respect to the product of two independent Schur functions becomes a tau function of the 2D Toda hierarchy. The associated Lax operators turn out to have a particular factorized form. This factorized form of the Lax operators characterizes a generalization of the Ablowitz-Ladik hierarchy embedded in the 2D Toda hierarchy. The generalized Ablowitz-Ladik hierarchy is thus identified as a fundamental integrable structure of topological string theory on the generalized conifolds.
\end{abstract}

Pacs Numbers: 02.30.Ik, 11.25.Hf

2010 Mathematics Subject Classification: 17B80, 35Q55, 81T30

Key words: topological string, generalized conifold, free fermion, quantum torus, quantum dilogarithm, Toda hierarchy, Ablowitz-Ladik hierarchy

\section{Introduction}

This paper presents an extension of our previous work [1] on the integrable structure of a modified "melting crystal model". The partition function of

\footnotetext{
*E-mail: takasaki@math.h.kyoto-u.ac.jp
} 
the previous model is a generating function of (equivariant) local GromovWitten invariants of the resolved conifold [2]. The relevance of the AblowitzLadik hierarchy [3, 4] (equivalently, the relativistic Toda hierarchy [5, 6, 7]) to this model was conjectured by Brini from an order-by-order analysis of the genus expansion of the partition function [8]. We deformed the modified melting crystal model by two sets of external potentials with coupling constants $\boldsymbol{t}=\left(t_{1}, t_{2}, \ldots\right)$ and $\overline{\boldsymbol{t}}=\left(\bar{t}_{1}, \bar{t}_{2}, \ldots\right)$, and employed the method [9, 10] developed for the genuine melting crystal model [11, 12] to show that the deformed partition function is essentially a tau function $\tau(s, \boldsymbol{t}, \overline{\boldsymbol{t}})$ of the $2 \mathrm{D}$ Toda hierarchy [13, 14. We further found that the associated Lax operators of the Toda hierarchy have a particular factorized form, which coincides with the condition that characterizes the Ablowitz-Ladik hierarchy as a reduction of the Toda hierarchy [15]. Thus the solution of the Toda hierarchy in question turns out to be a solution of the Ablowitz-Ladik hierarchy.

In the perspective of topological string theory, the tau function of the modified melting crystal model is a generating function of open string amplitudes on the resolved conifold. One can generalize it to toric Calabi-Yau threefolds whose toric diagrams are obtained by "triangulation of a strip". For such manifolds, the method of topological vertex [16] for calculating open string amplitudes simplifies drastically [17]. Namely, the amplitudes can be expressed as the matrix elements $\langle\lambda|g| \mu\rangle$ of an operator $g$ on the Fock space of $2 \mathrm{D}$ charged free fermion fields, where $\lambda$ and $\mu$ are partitions assigned to the leftmost and rightmost external edges of the web diagram, and $\langle\lambda|$ and $|\mu\rangle$ are normalized excited states labelled by these partitions [18, 19, 20]. The generating function of the matrix elements $\langle\lambda, s|g| \mu, s\rangle$ (extended to the charge- $s$ sectors of the Fock space) with respect to the product $s_{\lambda}(\boldsymbol{x}) s_{\mu}(\boldsymbol{y})$ of Schur functions of two independent sets of variables $\boldsymbol{x}, \boldsymbol{y}$ becomes a tau function $\tau(s, \boldsymbol{t}, \overline{\boldsymbol{t}})$ of the Toda hierarchy by a well known transformation of variables from $\boldsymbol{x}, \boldsymbol{y}$ to $\boldsymbol{t}, \overline{\boldsymbol{t}}[21$.

Among these generalizations to toric Calabi-Yau threefolds, we now consider the case where the web diagram consists of a zig-zag spine of $2 N-1$ internal edges and $2 N+2$ external edges emanating from the spine (see Fig. 11). The resolved conifold amounts to the case of $N=1$. For this "generalized conifold", we shall show that the associated Lax operators of the Toda hierarchy have the factorized form

$$
L=B e^{(1-N) \partial_{s}} C^{-1}, \quad \bar{L}^{-1}=D C e^{(N-1) \partial_{s}} B^{-1},
$$

where $e^{\partial_{s}}\left(\partial_{s}=\partial / \partial s\right)$ denotes the shift operator that acts on functions $\psi(s)$ of $s \in \mathbb{Z}$ as $e^{\partial_{s}} \psi(s)=\psi(s+1), B$ and $C$ are finite order difference operators 


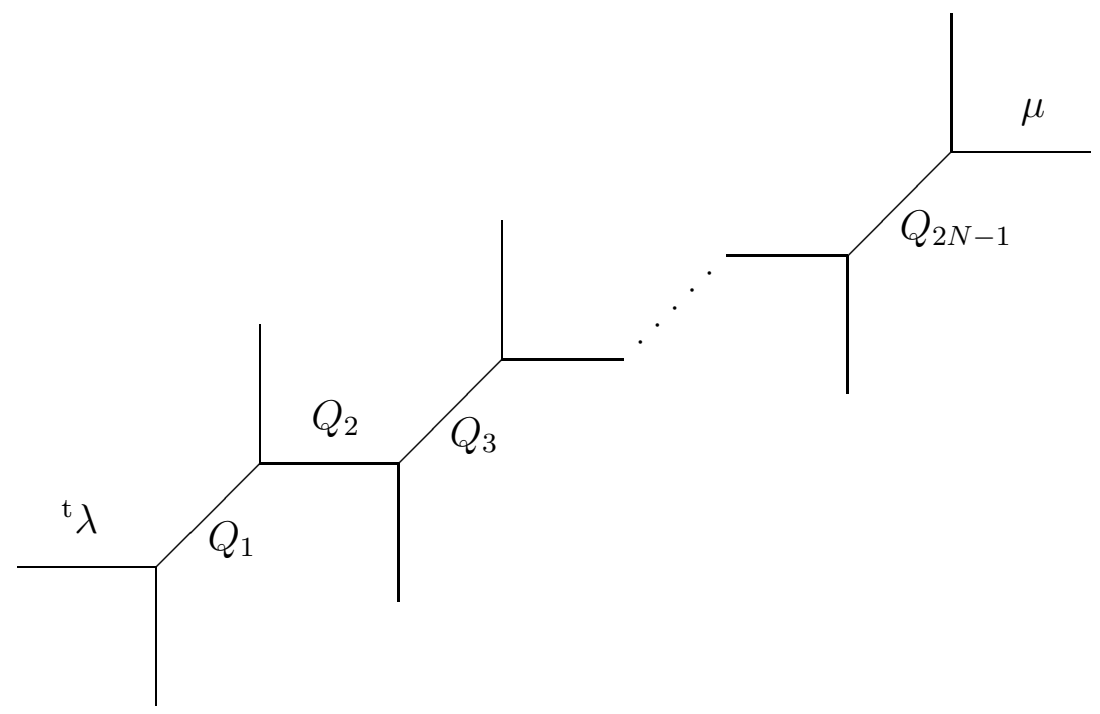

Figure 1: Web diagram of generalized conifold

of the form

$$
\begin{aligned}
& B=e^{N \partial_{s}}+b_{1}(s) e^{(N-1) \partial_{s}}+\cdots+b_{N}(s), \\
& C=1+c_{1}(s) e^{-\partial_{s}}+\cdots+c_{N}(s) e^{-N \partial_{s}},
\end{aligned}
$$

and $D$ is a constant. The factorized form (1) is preserved by the flows of the Toda hierarchy, hence defines a generalization of the Ablowitz-Ladik hierarchy as a subsystem of the Toda hierarchy. It is this generalized AblowitzLadik hierarchy that we identify as a fundamental integrable structure of topological string theory on the $N$-th generalized conifold.

To derive this result, we adopt the same strategy as in the previous work 11. Namely, we translate the fermionic construction of the tau function to the language of $\mathbb{Z} \times \mathbb{Z}$ matrices. The operator $g$ thus corresponds to an infinite matrix $U$. The associated solution of the Toda hierarchy can be captured by a factorization problem of infinite matrices [22, 23, 24]. Actually, infinite matrices arising therein are avatars of difference operators in the Lax formalism of the Toda hierarchy. The factors in the factorization problem amount to the dressing operators $W, \bar{W}$, which in turn determine the Lax operators $L, \bar{L}$. We can find an explicit form of these matrices at least at the initial time $\boldsymbol{t}=\overline{\boldsymbol{t}}=\mathbf{0}$. The initial values of $L$ and $\bar{L}^{-1}$ turns out to have the factorized form (1). This is enough to conclude that $L$ and $\bar{L}^{-1}$ are similarly factorized at all times, because the factorized form (1) is preserved by the flows of the Toda hierarchy. 
This paper is organized as follows. Section 2 reviews the fermionic expression of open string amplitudes and the associated tau function. The operator $g$ shows up here as a product of several building blocks. Section 3 shows a dictionary that translates the fermionic setting to the language of infinite matrices. The matrix $U$ is derived from $g$ by this dictionary. Section 4 introduces the matrix factorization problem that determines the dressing operators. The initial values of the dressing operators are explicitly calculated here. This result is used in Section 5 to calculate the initial values of the Lax operators. The initial values are shown to have the factorized form (1). Section 6 is devoted to the generalized Ablowitz-Ladik hierarchy. Fundamental properties, such as the consistency of this system, are proved here. As a corollary, the solution of the Toda hierarchy obtained from $U$ turns out to be a solution of the generalized Ablowitz-Ladik hierarchy. Section 7 presents our conclusion.

\section{Tau function in fermionic language}

Let $\psi_{n}$ and $\psi_{n}^{*}, n \in \mathbb{Z}$, be the Fourier modes of $2 \mathrm{D}$ charged free fermion fields 25

$$
\psi(z)=\sum_{n \in \mathbb{Z}} \psi_{n} z^{-n-1}, \quad \psi^{*}(z)=\sum_{n \in \mathbb{Z}} \psi_{n}^{*} z^{-n}
$$

They satisfy the anti-commutation relations

$$
\psi_{m} \psi_{n}^{*}+\psi_{n}^{*} \psi_{n}=\delta_{m+n, 0}, \quad \psi_{m} \psi_{n}+\psi_{n} \psi_{m}=0, \quad \psi_{m}^{*} \psi_{n}^{*}+\psi_{n}^{*} \psi_{m}^{*}=0
$$

The Fock and dual Fock space can be decomposed into the eigenspaces of the $U(1)$-charge operator

$$
J_{0}=\sum_{n \in \mathbb{Z}}: \psi_{-n} \psi_{n}^{*}
$$

Let $\langle s|$ and $|s\rangle, s \in \mathbb{Z}$, denote the ground states in the charge- $s$ sector:

$$
\left\langle s\left|=\left\langle-\infty\left|\cdots \psi_{s-1}^{*} \psi_{s}^{*}, \quad\right| s\right\rangle=\psi_{-s} \psi_{-s+1} \cdots\right|-\infty\right\rangle
$$

Normalized excited states are labelled by partitions $\lambda=\left(\lambda_{1}, \lambda_{2}, \ldots\right), \lambda_{1} \geq$ $\lambda_{2} \geq \cdots \geq 0$, as $\langle\lambda, s|$ and $|\lambda, s\rangle$ :

$$
\left\langle\lambda, s\left|=\left\langle-\infty\left|\cdots \psi_{\lambda_{2}+s-1}^{*} \psi_{\lambda_{1}+s}^{*}, \quad\right| \lambda, s\right\rangle=\psi_{-\lambda_{1}-s} \psi_{-\lambda_{2}-s+1} \cdots\right|-\infty\right\rangle .
$$


Building blocks of the fermionic expression of open string amplitudes are the special fermion bilinears

$$
\begin{gathered}
J_{k}=\sum_{n \in \mathbb{Z}}: \psi_{-n} \psi_{n+k}^{*}:, \quad k \in \mathbb{Z}, \\
L_{0}=\sum_{n \in \mathbb{Z}} n: \psi_{-n} \psi_{n}^{*}:, \quad W_{0}=\sum_{n \in \mathbb{Z}} n^{2}: \psi_{-n} \psi_{n}^{*}:
\end{gathered}
$$

and the vertex operators [26, 27]

$$
\Gamma_{ \pm}(z)=\exp \left(\sum_{k=1}^{\infty} \frac{z^{k}}{k} J_{ \pm k}\right), \quad \Gamma_{ \pm}^{\prime}(z)=\exp \left(-\sum_{k=1}^{\infty} \frac{(-z)^{k}}{k} J_{ \pm k}\right)
$$

It is convenient to define the multi-variate vertex operators $\Gamma_{ \pm}(\boldsymbol{x})$ and $\Gamma_{ \pm}^{\prime}(\boldsymbol{x})$, $\boldsymbol{x}=\left(x_{1}, x_{2}, \ldots\right)$, as well:

$$
\Gamma_{ \pm}(\boldsymbol{x})=\prod_{i=1}^{\infty} \Gamma_{ \pm}\left(x_{i}\right), \quad \Gamma_{ \pm}^{\prime}(\boldsymbol{x})=\prod_{i=1}^{\infty} \Gamma_{ \pm}^{\prime}\left(x_{i}\right) .
$$

These operators are "neutral" or "charge-preserving", i.e., do not mix different charge sectors. $L_{0}$ and $W_{0}$ are "diagonal" and have the $s$-dependent matrix elements

$$
\begin{aligned}
& \left\langle\lambda, s\left|L_{0}\right| \mu, s\right\rangle=\delta_{\lambda \mu}\left(|\lambda|+\frac{s(s+1)}{2}\right) \\
& \left\langle\lambda, s\left|W_{0}\right| \mu, s\right\rangle=\delta_{\lambda \mu}\left(\kappa(\lambda)+(2 s+1)|\lambda|+\frac{s(s+1)(2 s+1)}{6}\right),
\end{aligned}
$$

where $\kappa(\lambda)$ denotes the quadratic Casimir invariant

$$
\kappa(\lambda)=\sum_{i \geq 1} \lambda_{i}\left(\lambda_{i}-2 i+1\right)=\sum_{i \geq 1}\left(\left(\lambda_{i}-i+1 / 2\right)^{2}-(-i+1 / 2)^{2}\right)
$$

of the partition $\lambda=\left(\lambda_{1}, \lambda_{2}, \ldots\right)$. The matrix elements of $\Gamma_{ \pm}(\boldsymbol{x})$ and $\Gamma_{ \pm}^{\prime}(\boldsymbol{x})$ are independent of the charge sector, and become skew Schur functions [25, 28]:

$$
\begin{gathered}
\left\langle\lambda, s\left|\Gamma_{-}(\boldsymbol{x})\right| \mu, s\right\rangle=\left\langle\mu, s\left|\Gamma_{+}(\boldsymbol{x})\right| \lambda, s\right\rangle=s_{\lambda / \mu}(\boldsymbol{x}), \\
\left\langle\lambda, s\left|\Gamma_{-}^{\prime}(\boldsymbol{x})\right| \mu, s\right\rangle=\left\langle\mu, s\left|\Gamma_{+}^{\prime}(\boldsymbol{x})\right| \lambda, s\right\rangle=s{ }_{\lambda /{ }^{\mathrm{t}} \mu}(\boldsymbol{x}),
\end{gathered}
$$

where ${ }^{\mathrm{t}} \lambda$ and ${ }^{\mathrm{t}} \mu$ denote the conjugate (or transposed) partitions of $\lambda$ and $\mu$. 
$J_{k}$ 's play a fundamental role in the fermionic expression of tau functions of the Toda hierarchy as well [29]. General tau functions can be expressed as

$$
\tau(s, \boldsymbol{t}, \overline{\boldsymbol{t}})=\left\langle s\left|\exp \left(\sum_{k=1}^{\infty} t_{k} J_{k}\right) g \exp \left(-\sum_{k=1}^{\infty} \bar{t}_{k} J_{-k}\right)\right| s\right\rangle,
$$

where $g$ is an element of the Clifford group $\widehat{\mathrm{GL}}(\infty)$ [25] generated by the exponentials $e^{\hat{X}}$ of neutral fermion bilinears

$$
\hat{X}=\sum_{m, n \in \mathbb{Z}} x_{m n}: \psi_{-m} \psi_{n}^{*}
$$

The tau function of the modified melting crystal model [1] is obtained from the special operator

$$
g=q^{W_{0} / 2} \Gamma_{-}\left(q^{-\rho}\right) \Gamma_{+}\left(q^{-\rho}\right) Q^{L_{0}} \Gamma_{-}^{\prime}\left(q^{-\rho}\right) \Gamma_{+}^{\prime}\left(q^{-\rho}\right) q^{-W_{0} / 2},
$$

where $q$ and $Q$ are positive constants, $q$ is restricted to the range $0<q<1$, and $\Gamma_{ \pm}\left(q^{-\rho}\right)$ and $\Gamma_{ \pm}^{\prime}\left(q^{-\rho}\right)$ are the specialization of $\Gamma_{ \pm}(\boldsymbol{x})$ and $\Gamma_{ \pm}^{\prime}(\boldsymbol{x})$ to

$$
q^{-\rho}=\left(q^{1 / 2}, q^{3 / 2}, \ldots, q^{k+1 / 2}, \ldots\right) .
$$

Topological string theory on the $N$-th generalized conifold is related to the following generalization of (8) [19, 20]:

$$
\begin{aligned}
g= & q^{W_{0} / 2} \Gamma_{-}\left(q^{-\rho}\right) \Gamma_{+}\left(q^{-\rho}\right) Q_{1}^{L_{0}} \Gamma_{-}^{\prime}\left(q^{-\rho}\right) \Gamma_{+}^{\prime}\left(q^{-\rho}\right) Q_{2}^{L_{0}} \\
& \times \Gamma_{-}\left(q^{-\rho}\right) \Gamma_{+}\left(q^{-\rho}\right) Q_{3}^{L_{0}} \Gamma_{-}^{\prime}\left(q^{-\rho}\right) \Gamma_{+}^{\prime}\left(q^{-\rho}\right) Q_{4}^{L_{0}} \\
& \times \cdots \\
& \times \Gamma_{-}\left(q^{-\rho}\right) \Gamma_{+}\left(q^{-\rho}\right) Q_{2 N-1}^{L_{0}} \Gamma_{-}^{\prime}\left(q^{-\rho}\right) \Gamma_{+}^{\prime}\left(q^{-\rho}\right) q^{-W_{0} / 2} .
\end{aligned}
$$

$Q_{1}, \ldots, Q_{2 N-1}$ are "Kähler parameters" assigned to the internal edges of the web diagram (see Fig. 1). The matrix elements $\langle\lambda|g| \mu\rangle$ of $g$ in the Fock space give a compact expression of the open string amplitudes $Z_{\mathrm{t}_{\lambda} \emptyset \ldots \emptyset \mu}$ in the setting where the leftmost and rightmost external edges are assigned with the non-trivial partitions ${ }^{\mathrm{t}} \lambda, \mu$ whereas the other external edges are given the zero partition $\emptyset 1$.

The logarithm of $Z_{{ }_{\lambda} \emptyset \ldots \emptyset \mu}$ is a generating functions of all genus local Gromov-Witten invariants with respect to the parameters $q, Q_{1}, \ldots, Q_{2 N-1}$.

\footnotetext{
${ }^{1}$ As we show in Appendix A, the matrix elements $\langle\lambda|g| \mu\rangle$ are slightly different from the true amplitudes $Z_{\mathrm{t}} \lambda \emptyset \ldots \emptyset_{\mu}$. The difference, however, is rather immaterial and does not affect our consideration on the integrable structure.
} 
The partitions specify the topological type (i.e., winding numbers) of boundaries of the string world sheet. One can assign non-trivial partitions $\beta_{1}, \ldots, \beta_{2 N}$ to the intermediate external edges to define more general amplitudes $Z_{\mathrm{t}_{\lambda} \beta_{1} \ldots \beta_{2 N} \mu}$ as well. We refer a precise definition of these notions to Mariño's book [30]. The fermionic expression of these amplitudes can be derived from a combinatorial expression obtained by the method of topological vertex (see Appendix A).

The matrix elements $\langle\lambda|g| \mu\rangle$ and their extensions $\langle\lambda, s|g| \mu, s\rangle$ to the charge$s$ sectors can be assembled to the generating functions

$$
\sum_{\lambda, \mu \in \mathcal{P}} s_{\lambda}(\boldsymbol{x})\langle\lambda, s|g| \mu, s\rangle s_{\mu}(\boldsymbol{y})
$$

where $\mathcal{P}$ is the set of all partitions, and $s_{\lambda}(\boldsymbol{x})$ and $s_{\mu}(\boldsymbol{y})$ are the Schur functions of multi-variables $\boldsymbol{x}=\left(x_{1}, x_{2}, \ldots\right)$ and $\boldsymbol{y}=\left(y_{1}, y_{2}, \ldots\right)$. These generating functions become the tau function (7) by changing variables from $(\boldsymbol{x}, \boldsymbol{y})$ to $(\boldsymbol{t}, \overline{\boldsymbol{t}})$ as

$$
t_{k}=\frac{1}{k} \sum_{i \geq 1} x_{i}^{k}, \quad \bar{t}_{k}=-\frac{1}{k} \sum_{i \geq 1} y_{i}^{k} .
$$

\section{Translation to infinite matrices}

The foregoing fermionic setting can be translated to the language of infinite matrices. In particular, we obtain a matrix that represents the element (9) of $\widehat{\mathrm{GL}}(\infty)$. We shall use this matrix to characterize the associated solution of the Toda hierarchy in the Lax formalism.

It is well known that neutral fermion bilinears are in one-to-one correspondence with $\mathbb{Z} \times \mathbb{Z}$ matrices [25]:

$$
\hat{X} \longleftrightarrow X=\sum_{m, n \in \mathbb{Z}} x_{m n} E_{m n}, \quad E_{m n}=\left(\delta_{i m} \delta_{j n}\right)_{i, j \in \mathbb{Z}}
$$

Apart from c-number corrections, they have the same Lie algebraic structure, namely,

$$
[\hat{X}, \hat{Y}]=\widehat{[X, Y]}+c(X, Y)
$$

where $c(X, Y)$ is a c-number cocycle of $\mathrm{gl}(\infty)$. Thus fermion bilinears form a central extension $\widehat{\mathrm{gl}}(\infty)$ of $\mathrm{gl}(\infty)$.

Actually, the matrix representation gives us more freedom, because the set of infinite matrices is equipped with multiplication as well as Lie brackets. For 
example, matrix representation of $L_{0}, M_{0}, J_{k}$ (let us use the same notations as fermion bilinears) can be written as

$$
L_{0}=\Delta, \quad W_{0}=\Delta^{2}, \quad J_{k}=\Lambda^{k},
$$

where

$$
\Delta=\sum_{n \in \mathbb{Z}} n E_{n n}, \quad \Lambda=\sum_{n \in \mathbb{Z}} E_{n, n+1}
$$

Note that $\Lambda$ and $\Delta$ amount to the shift operator $e^{\partial_{s}}$ and the multiplication operator $s$ on the $1 \mathrm{D}$ lattice $\mathbb{Z}$ :

$$
\Lambda \longleftrightarrow e^{\partial_{s}}, \quad \Delta \longleftrightarrow s
$$

The matrix representation of fermion bilinears can be lifted up to $\widehat{\mathrm{GL}}(\infty)$ [25]. By definition, the matrix representation $A=\left(a_{i j}\right)$ of $g \in \widehat{\mathrm{GL}}(\infty)$ is determined by the Bogoliubov transformation on the linear span of $\psi_{n}$ 's:

$$
g \psi_{n} g^{-1}=\sum_{m \in \mathbb{Z}} \psi_{m} a_{m n}
$$

If $g$ is the exponential $e^{\hat{X}}$ of a neutral fermion bilinear $\hat{X}, A$ is the exponential $e^{X}$ of the matrix $X$. We can thereby find, from (12), an explicit form of the matrix representation of building blocks in (9):

(i) The matrix representation of $\Gamma_{ \pm}(z)$ and $\Gamma_{ \pm}^{\prime}(z)$, denoted by the same notations, reads

$$
\begin{aligned}
& \Gamma_{ \pm}(z)=\exp \left(\sum_{k=1}^{\infty} \frac{z^{k}}{k} \Lambda^{ \pm k}\right)=\left(1-z \Lambda^{ \pm 1}\right)^{-1}, \\
& \Gamma_{ \pm}^{\prime}(z)=\exp \left(-\sum_{k=1}^{\infty} \frac{(-z)^{k}}{k} \Lambda^{ \pm k}\right)=1+z \Lambda^{ \pm} .
\end{aligned}
$$

Consequently, $\Gamma_{ \pm}\left(q^{-\rho}\right)$ and $\Gamma_{ \pm}^{\prime}\left(q^{-\rho}\right)$ become infinite products of matrices:

$$
\begin{aligned}
& \Gamma_{ \pm}\left(q^{-\rho}\right)=\prod_{i=1}^{\infty}\left(1-q^{i-1 / 2} \Lambda^{ \pm 1}\right)^{-1} \\
& \Gamma_{ \pm}^{\prime}\left(q^{-\rho}\right)=\prod_{i=1}^{\infty}\left(1+q^{i-1 / 2} \Lambda^{ \pm 1}\right)
\end{aligned}
$$

These infinite products may be thought of as matrix-valued "quantum dilogarithm" [31, 32]. 
(ii) $q^{W_{0} / 2}$ and $Q^{L_{0}}$ become the diagonal matrices

$$
q^{\Delta^{2} / 2}=\sum_{n \in \mathbb{Z}} q^{n^{2} / 2} E_{n n}, \quad Q^{\Delta}=\sum_{n \in \mathbb{Z}} Q^{n} E_{n n} .
$$

Thus the following matrix representation of (9) is obtained:

$$
\begin{aligned}
U= & q^{\Delta^{2} / 2} \Gamma_{-}\left(q^{-\rho}\right) \Gamma_{+}\left(q^{-\rho}\right) Q_{1}^{\Delta} \Gamma_{-}^{\prime}\left(q^{-\rho}\right) \Gamma_{+}^{\prime}\left(q^{-\rho}\right) Q_{2}^{\Delta} \\
& \times \Gamma_{-}\left(q^{-\rho}\right) \Gamma_{+}\left(q^{-\rho}\right) Q_{3}^{\Delta} \Gamma_{-}^{\prime}\left(q^{-\rho}\right) \Gamma_{+}^{\prime}\left(q^{-\rho}\right) Q_{4}^{\Delta} \\
& \times \cdots \\
& \times \Gamma_{-}\left(q^{-\rho}\right) \Gamma_{+}\left(q^{-\rho}\right) Q_{2 N-1}^{\Delta} \Gamma_{-}^{\prime}\left(q^{-\rho}\right) \Gamma_{+}^{\prime}\left(q^{-\rho}\right) q^{-\Delta^{2} / 2}
\end{aligned}
$$

Let us make several technical remarks:

Remark 1. $Q^{\Delta}$ and $\Lambda^{k}$ 's satisfy the commutation relation

$$
\Lambda^{k} Q^{\Delta}=Q^{k} Q^{\Delta} \Lambda^{k}
$$

The order of $\Gamma_{ \pm}, \Gamma_{ \pm}^{\prime}$ and $Q^{\Delta}$ 's in (16) can be thereby exchanged as

$$
\begin{aligned}
& \Gamma_{ \pm}\left(q^{-\rho}\right) Q^{\Delta}=Q^{\Delta} \Gamma_{ \pm}\left(Q^{ \pm 1} q^{-\rho}\right), \\
& \Gamma_{ \pm}^{\prime}\left(q^{-\rho}\right) Q^{\Delta}=Q^{\Delta} \Gamma_{ \pm}^{\prime}\left(Q^{ \pm 1} q^{-\rho}\right) .
\end{aligned}
$$

Remark 2. (14) can be slightly generalized as

$$
\begin{aligned}
& \Gamma_{ \pm}\left(Q q^{-\rho}\right)=\prod_{i=1}^{\infty}\left(1-Q q^{i-1 / 2} \Lambda^{ \pm 1}\right)^{-1} \\
& \Gamma_{ \pm}^{\prime}\left(Q q^{-\rho}\right)=\prod_{i=1}^{\infty}\left(1+Q q^{i-1 / 2} \Lambda^{ \pm 1}\right) .
\end{aligned}
$$

Remark 3. $q^{\Delta^{2} / 2}$ transforms $\Lambda^{k}$ by conjugation as

$$
q^{\Delta^{2} / 2} \Lambda^{k} q^{-\Delta^{2} / 2}=q^{-k \Delta-k^{2} / 2} \Lambda^{k} .
$$

This is a special case of "the third shift symmetry" in the terminology of our previous work [1]. $\Lambda^{k}$ and $q^{-k \Delta-k^{2} / 2} \Lambda^{k}$ are elements of the quantum torus algebra spanned by

$$
v_{m}^{(k)}=q^{k m / 2} q^{k \Delta} \Lambda^{m}=q^{-k m / 2} \Lambda^{m} q^{k \Delta}, \quad k, m \in \mathbb{Z} .
$$




\section{Initial values of dressing operators}

Given the infinite matrix $U$ representing an element $g$ of $\widehat{\mathrm{GL}}(\infty) 2$, the associated solution of the Toda hierarchy can be characterized by the factorization problem [22, 23, 24]

$$
\exp \left(\sum_{k=1}^{\infty} t_{k} \Lambda^{k}\right) U \exp \left(-\sum_{k=1}^{\infty} \bar{t}_{k} \Lambda^{-k}\right)=W^{-1} \bar{W}
$$

where $W$ is a lower triangular matrix with all diagonal elements being equal to 1 , and $\bar{W}$ is an upper triangular matrix with all diagonal elements being non-zero.

$W$ and $\bar{W}$ play the role of dressing operators. As matrix-valued functions of $\boldsymbol{t}$ and $\overline{\boldsymbol{t}}$, they satisfy the Sato equations

$$
\begin{array}{ll}
\frac{\partial W}{\partial t_{k}}=-\left(W \Lambda^{k} W^{-1}\right)_{<0} W, & \frac{\partial W}{\partial \bar{t}_{k}}=\left(\bar{W} \Lambda^{-k} \bar{W}^{-1}\right)_{<0} W, \\
\frac{\partial \bar{W}}{\partial t_{k}}=\left(W \Lambda^{k} W^{-1}\right)_{\geq 0} \bar{W}, & \frac{\partial \bar{W}}{\partial \bar{t}_{k}}=-\left(\bar{W} \Lambda^{-k} \bar{W}^{-1}\right)_{\geq 0} \bar{W},
\end{array}
$$

where $(\quad)_{\geq 0}$ and $(\quad)_{<0}$ denote the projection to the upper and strictly lower triangular parts, i.e.,

$$
\left(\sum_{m, n} a_{m n} E_{m n}\right)_{\geq 0}=\sum_{m \leq n} a_{m n} E_{m n},\left(\sum_{m, n} a_{m n} E_{m n}\right)_{<0}=\sum_{m>n} a_{m n} E_{m n} .
$$

The Lax operators are obtained by "dressing" the shift matrix $\Lambda$ as

$$
L=W \Lambda W^{-1}, \quad \bar{L}=\bar{W} \Lambda \bar{W},
$$

and satisfy the Lax equations

$$
\begin{array}{ll}
\frac{\partial L}{\partial t_{k}}=\left[\left(L^{k}\right)_{\geq 0}, L\right], & \frac{\partial L}{\partial \bar{t}_{k}}=\left[\left(\bar{L}^{-k}\right)_{<0}, L\right] \\
\frac{\partial \bar{L}}{\partial t_{k}}=\left[\left(L^{k}\right)_{\geq 0}, \bar{L}\right], & \frac{\partial \bar{L}}{\partial \bar{t}_{k}}=\left[\left(\bar{L}^{-k}\right)_{<0}, \bar{L}\right] .
\end{array}
$$

Note that the inverse matrix $\bar{L}^{-1}$, too, satisfies Lax equations of the same form

$$
\frac{\partial \bar{L}^{-1}}{\partial t_{k}}=\left[\left(L^{k}\right)_{\geq 0}, \bar{L}^{-1}\right], \quad \frac{\partial \bar{L}^{-1}}{\partial \bar{t}_{k}}=\left[\left(\bar{L}^{-k}\right)_{<0}, \bar{L}^{-1}\right]
$$

\footnotetext{
${ }^{2}$ To give a solution of the Toda hierarchy, $g$ has to satisfy a set of genericity conditions, e.g., $\langle s|g| s\rangle \neq 0$ for all $s \in \mathbb{Z}[29]$.
} 
Actually, it is $\bar{L}^{-1}$ rather than $\bar{L}$ that plays a fundamental role in our consideration on the solution obtained from (16).

In a general case, solving the factorization problem (22) directly is extremely difficult. In the case of (16), however, we can find an explicit form of the solution at the initial time $\boldsymbol{t}=\overline{\boldsymbol{t}}=\mathbf{0}$ as follows.

Proposition 1. The matrix (16) can be factorized as

$$
U=W_{(0)}^{-1} \bar{W}_{(0)},
$$

where

$$
\begin{aligned}
W_{(0)}= & q^{\Delta^{2} / 2} \cdot \prod_{n=1}^{N} \Gamma_{-}\left(Q^{(2 n-1)} q^{-\rho}\right)^{-1} \Gamma_{-}^{\prime}\left(Q^{(2 n)} q^{-\rho}\right)^{-1} \cdot q^{-\Delta^{2} / 2}, \\
\bar{W}_{(0)}= & q^{\Delta^{2} / 2} \cdot \prod_{n=1}^{N} \Gamma_{+}\left(Q^{(2 n-1)-1} q^{-\rho}\right) \Gamma_{+}^{\prime}\left(Q^{(2 n)-1} q^{-\rho}\right) \\
& \times\left(Q_{1} \cdots Q_{2 N-1}\right)^{\Delta} q^{-\Delta^{2} / 2},
\end{aligned}
$$

and

$$
Q^{(n)}=Q_{1} \cdots Q_{n-1}, \quad Q^{(1)}=1 .
$$

The factors $W_{(0)}, \bar{W}_{(0)}$ are the initial values $\left.W\right|_{\boldsymbol{t}=\overline{\boldsymbol{t}}=\mathbf{0}},\left.\bar{W}\right|_{\boldsymbol{t}=\overline{\boldsymbol{t}}=\mathbf{0}}$ of the dressing matrices for the solution of the Toda hierarchy obtained from (16).

Proof. Use the commutation relation (18) repeatedly to move $Q_{1}^{\Delta}, \ldots, Q_{2 N-1}^{\Delta}$ in (16) to the front of $q^{-\Delta^{2} / 2}$ as

$$
\begin{aligned}
U= & \left.q^{\Delta^{2} / 2} \Gamma_{-}\left(q^{-\rho}\right) \Gamma_{+}\left(q^{-\rho}\right) \Gamma_{-}^{\prime}\left(Q_{1} q^{-\rho}\right)\right) \Gamma_{+}^{\prime}\left(Q_{1}^{-1} q^{-\rho}\right) \\
& \times \Gamma_{-}\left(Q^{(3)} q^{-\rho}\right) \Gamma_{+}\left(Q^{(3)-1} q^{-\rho}\right) \Gamma_{-}^{\prime}\left(Q^{(4)} q^{-\rho}\right) \Gamma_{+}^{\prime}\left(Q^{(4)-1} q^{-\rho}\right) \\
& \times \cdots \\
& \times \Gamma_{-}\left(Q^{(2 N-1)} q^{-\rho}\right) \Gamma_{+}\left(Q^{(2 N-1)-1} q^{-\rho}\right) \Gamma_{-}^{\prime}\left(Q^{(2 N)} q^{-\rho}\right) \Gamma_{+}^{\prime}\left(Q^{(2 N)-1} q^{-\rho}\right) \\
& \times\left(Q_{1} \cdots Q_{2 N-1}\right)^{\Delta} q^{-\Delta^{2} / 2} .
\end{aligned}
$$

Since the vertex operators in the matrix representation (13) commute with each other, $\Gamma_{-}$'s and $\Gamma_{-}^{\prime}$ 's can be moved to the left side of $\Gamma_{+}$'s and $\Gamma_{+}^{\prime}$ 's. Thus $U$ can be converted to the factorized form with factors (26) and (27). Since (26) is a lower triangular matrix with unit diagonal elements and (27) is an upper triangular matrix with non-zero diagonal elements, these matrices are indeed the initial values of the dressing operators. 


\section{$5 \quad$ Initial values of Lax operators}

Let us calculate the initial values

$$
L_{(0)}=W_{(0)} \Lambda W_{(0)}^{-1}, \quad \bar{L}_{(0)}^{-1}=\bar{W}_{(0)} \Lambda^{-1} \bar{W}_{(0)}^{-1}
$$

of the Lax operators at $\boldsymbol{t}=\overline{\boldsymbol{t}}=\mathbf{0}$ from the explicit expressions (26) and (27) of the initial values of the dressing operators. As it turns out, they are non-commutative rational functions of $q^{\Delta}$ and $\Lambda$.

Plugging the explicit form of $W_{(0)}$ and $\bar{W}_{(0)}$ into (28) yields the following somewhat complicated expressions of $L_{(0)}$ and $\bar{L}_{(0)}^{-1}$ :

$$
\begin{aligned}
L_{(0)}= & q^{\Delta^{2} / 2} \prod_{n=1}^{N} \Gamma_{-}\left(Q^{(2 n-1)} q^{-\rho}\right)^{-1} \Gamma_{-}^{\prime}\left(Q^{(2 n)} q^{-\rho}\right)^{-1} \\
& \times q^{-\Delta^{2} / 2} \Lambda q^{\Delta^{2} / 2} \\
& \times \prod_{n=1}^{N} \Gamma_{-}^{\prime}\left(Q^{(2 n)} q^{-\rho}\right) \Gamma_{-}\left(Q^{(2 n-1)} q^{-\rho}\right) \cdot q^{-\Delta^{2} / 2} \\
\bar{L}_{(0)}^{-1}= & q^{\Delta^{2} / 2} \prod_{n=1}^{N} \Gamma_{+}\left(Q^{(2 n-1)-1} q^{-\rho}\right) \Gamma_{+}^{\prime}\left(Q^{(2 n)-1} q^{-\rho}\right) \\
\times & \left(Q_{1} \cdots Q_{2 N-1}\right)^{\Delta} q^{-\Delta^{2} / 2} \Lambda^{-1} q^{\Delta^{2} / 2}\left(Q_{1} \cdots Q_{2 N-1}\right)^{-\Delta} \\
& \times \prod_{n=1}^{N} \Gamma_{+}^{\prime}\left(Q^{(2 n)-1} q^{-\rho}\right)^{-1} \Gamma_{+}\left(Q^{(2 n-1)-1} q^{-\rho}\right)^{-1} \cdot q^{-\Delta^{2} / 2} .
\end{aligned}
$$

We can use (20) and (17) to rewrite the products in the middle of the right hand side as

$$
\begin{gathered}
q^{-\Delta^{2} / 2} \Lambda q^{-\Delta^{2} / 2}=q^{\Delta+1 / 2} \Lambda, \\
\left(Q_{1} \cdots Q_{2 N-1}\right)^{\Delta} q^{-\Delta^{2} / 2} \Lambda^{-1} q^{\Delta^{2} / 2}\left(Q_{1} \cdots Q_{2 N-1}\right)^{-\Delta}=Q_{1} \cdots Q_{2 N-1} q^{-\Delta+1 / 2} \Lambda^{-1} .
\end{gathered}
$$

Since the last factors $\Lambda^{ \pm 1}$ in the right hand side commute with $\Gamma^{\prime}$ s and $\Gamma^{\prime}$ s, let us move them to the front of $q^{-\Delta^{2} / 2}$ as

$$
\begin{aligned}
L_{(0)}= & q^{\Delta^{2} / 2} \prod_{n=1}^{N} \Gamma_{-}\left(Q^{(2 n-1)} q^{-\rho}\right)^{-1} \Gamma_{-}^{\prime}\left(Q^{(2 n)} q^{-\rho}\right)^{-1} \cdot q^{\Delta+1 / 2} \\
& \times \prod_{n=1}^{N} \Gamma_{-}^{\prime}\left(Q^{(2 n)} q^{-\rho}\right) \Gamma_{-}\left(Q^{(2 n-1)} q^{-\rho}\right) \cdot \Lambda q^{-\Delta^{2} / 2}
\end{aligned}
$$


and

$$
\begin{aligned}
\bar{L}_{(0)}^{-1}= & Q_{1} \cdots Q_{2 N-1} q^{\Delta^{2} / 2} \prod_{n=1}^{N} \Gamma_{+}\left(Q^{(2 n-1)-1} q^{-\rho}\right) \Gamma_{+}^{\prime}\left(Q^{(2 n)-1} q^{-\rho}\right) \cdot q^{-\Delta+1 / 2} \\
& \times \prod_{n=1}^{N} \Gamma_{+}^{\prime}\left(Q^{(2 n)-1} q^{-\rho}\right)^{-1} \Gamma_{+}\left(Q^{(2 n-1)-1} q^{-\rho}\right)^{-1} \cdot \Lambda^{-1} q^{-\Delta^{2} / 2}
\end{aligned}
$$

To simplify these expressions, we use the following identities that underlie the "shift symmetries" of the quantum torus algebra [9, 10, 1].

\section{Lemma 1.}

$$
\begin{aligned}
\Gamma_{-}\left(Q q^{-\rho}\right)^{-1} q^{\Delta} \Gamma_{-}\left(Q q^{-\rho}\right) & =q^{\Delta}\left(1-Q q^{-1 / 2} \Lambda^{-1}\right), \\
\Gamma_{-}^{\prime}\left(Q q^{-\rho}\right)^{-1} q^{\Delta} \Gamma_{-}^{\prime}\left(Q q^{-\rho}\right) & =q^{\Delta}\left(1+Q q^{-1 / 2} \Lambda^{-1}\right)^{-1}, \\
\Gamma_{+}\left(Q^{-1} q^{-\rho}\right) q^{-\Delta} \Gamma_{+}\left(Q^{-1} q^{-\rho}\right)^{-1} & =q^{-\Delta}\left(1-Q^{-1} q^{-1 / 2} \Lambda\right)^{-1}, \\
\Gamma_{+}^{\prime}\left(Q^{-1} q^{-\rho}\right) q^{-\Delta} \Gamma_{+}^{\prime}\left(Q^{-1} q^{-\rho}\right)^{-1} & =q^{-\Delta}\left(1+Q^{-1} q^{-1 / 2} \Lambda\right) .
\end{aligned}
$$

Proof. Use the commutation relations

$$
\Lambda^{k} q^{\Delta}=q^{k} q^{\Delta} \Lambda^{k}
$$

(which is part of the structure of the quantum torus algebra) to exchange the order of $\Gamma_{-}\left(Q q^{-\rho}\right)$ and $q^{\Delta}$ as

$$
\Gamma_{-}\left(Q q^{-\rho}\right)^{-1} q^{\Delta} \Gamma_{-}\left(Q q^{-\rho}\right)=q^{\Delta} \Gamma_{-}\left(Q q^{-1} q^{-\rho}\right)^{-1} \Gamma_{-}\left(Q q^{-\rho}\right),
$$

and plug the infinite product expression (19) herein. The outcome is

$$
q^{\Delta} \prod_{i=1}^{\infty}\left(1-Q q^{i-3 / 2} \Lambda^{-1}\right) \cdot \prod_{i=1}^{\infty}\left(1-Q q^{i-1 / 2} \Lambda^{-1}\right)^{-1}=q^{\Delta}\left(1-Q q^{-1 / 2} \Lambda^{-1}\right) .
$$

Thus the first identity of (131) is obtained. The other identities can be derived in the same way.

The foregoing expressions (29) $-(30)$ of $L_{(0)}$ and $\bar{L}_{(0)}^{-1}$ can be simplified by the identities (31) as

$$
L_{(0)}=q^{\Delta^{2} / 2} q^{\Delta+1 / 2} \prod_{n=1}^{N}\left(1-Q^{(2 n-1)} q^{-1 / 2} \Lambda^{-1}\right)\left(1+Q^{(2 n)} q^{-1 / 2} \Lambda^{-1}\right)^{-1} \cdot \Lambda q^{-\Delta^{2} / 2}
$$


and

$$
\begin{aligned}
\bar{L}_{(0)}^{-1}= & Q_{1} \cdots Q_{2 N-1} q^{\Delta^{2} / 2} q^{-\Delta+1 / 2} \\
& \times \prod_{n=1}^{N}\left(1-Q^{(2 n-1)-1} q^{-1 / 2} \Lambda\right)^{-1}\left(1+Q^{(2 n)-1} q^{-1 / 2} \Lambda\right) \cdot \Lambda^{-1} q^{-\Delta^{2} / 2} .
\end{aligned}
$$

Lastly, we use (20) and (32) to convert (33) and (34) to rational functions of $q^{\Delta}$ and $\Lambda$ as

$$
\begin{aligned}
L_{(0)} & =q^{\Delta+1 / 2} \prod_{n=1}^{N}\left(1-Q^{(2 n-1)} q^{\Delta-1} \Lambda^{-1}\right)\left(1+Q^{(2 n)} q^{\Delta-1} \Lambda^{-1}\right)^{-1} \cdot q^{-\Delta-1 / 2} \Lambda \\
& =\prod_{n=1}^{N}\left(1-Q^{(2 n-1)} q^{\Delta} \Lambda^{-1}\right)\left(1+Q^{(2 n)} q^{\Delta} \Lambda^{-1}\right)^{-1} \cdot \Lambda
\end{aligned}
$$

and

$$
\begin{aligned}
\bar{L}_{(0)}^{-1}= & Q_{1} \cdots Q_{2 N-1} q^{-\Delta+1 / 2} \\
& \times \prod_{n=1}^{N}\left(1-Q^{(2 n-1)-1} q^{-\Delta-1} \Lambda\right)^{-1}\left(1+Q^{(2 n)-1} q^{-\Delta-1} \Lambda\right) \cdot q^{\Delta-1 / 2} \Lambda^{-1} \\
= & Q_{1} \cdots Q_{2 N-1} \prod_{n=1}^{N}\left(1-Q^{(2 n-1)-1} q^{-\Delta} \Lambda\right)^{-1}\left(1+Q^{(2 n)-1} q^{-\Delta} \Lambda\right) \cdot \Lambda^{-1}
\end{aligned}
$$

Although this is an almost final form, it will be suggestive to rewrite these expressions to the following "symmetric" form with the aid of (32).

Proposition 2. The initial values (28) of the Lax operators have the factorized form

$$
\begin{aligned}
& L_{(0)}=\prod_{n=1}^{N}\left(1-Q^{(2 n-1)} q^{\Delta} \Lambda^{-1}\right) \cdot \Lambda \cdot \prod_{n=1}\left(1+Q^{(2 n)} \Lambda^{-1} q^{\Delta}\right)^{-1}, \\
& \bar{L}_{(0)}^{-1}=Q_{1} \cdots Q_{2 N-1} \prod_{n=1}^{N}\left(1+Q^{(2 n)-1} q^{-\Delta} \Lambda\right) \cdot \Lambda^{-1} \cdot \prod_{n=1}^{N}\left(1-Q^{(2 n-1)-1} \Lambda q^{-\Delta}\right)^{-1} .
\end{aligned}
$$

To make contact with a generalization of the Ablowitz-Ladik hierarchy, let us restate this result in a slightly different form. The fully factorized form (37) of $L_{(0)}$ can be reassembled as

$$
L_{(0)}=B_{(0)} \Lambda^{1-N} C_{(0)}^{-1},
$$


where

$$
\begin{aligned}
& B_{(0)}=\prod_{n=1}^{N}\left(1-Q^{(2 n-1)} q^{\Delta} \Lambda^{-1}\right) \cdot \Lambda^{N}, \\
& C_{(0)}=\prod_{n=1}^{N}\left(1+Q^{(2 n)} \Lambda^{-1} q^{\Delta}\right) .
\end{aligned}
$$

Moreover, since

$$
\begin{gathered}
\prod_{n=1}^{N}\left(1+Q^{(2 n)-1} q^{-\Delta} \Lambda\right)=Q^{(2)-1} Q^{(4)-1} \cdots Q^{(2 N)-1} C\left(q^{-\Delta} \Lambda\right)^{N} \\
\prod_{n=1}^{N}\left(1-Q^{(2 n-1)-1} \Lambda q^{-\Delta}\right)^{-1}=Q^{(1)} Q^{(3)} \cdots Q^{(2 N-1)}\left(-\Lambda q^{-\Delta}\right)^{-N} \Lambda^{N} B^{-1}
\end{gathered}
$$

and

$$
\left(q^{-\Delta} \Lambda\right)^{N} \Lambda^{-1}\left(-\Lambda q^{-\Delta}\right)^{-N} \Lambda^{N}=(-1)^{N} \Lambda^{N-1},
$$

the fully factorized form (38) of $\bar{L}_{(0)}^{-1}$, too, can be cast into the similar expression

$$
\bar{L}_{(0)}^{-1}=D C_{(0)} \Lambda^{N-1} B_{(0)}^{-1},
$$

where

$$
D=(-1)^{N} Q_{1} \cdots Q_{2 N-1} \frac{Q^{(1)} Q^{(3)} \cdots Q^{(2 N-1)}}{Q^{(2)} Q^{(4)} \cdots Q^{(2 N)}}=(-1)^{N} Q_{2} Q_{4} \cdots Q_{2 N-2} .
$$

$B_{(0)}$ and $C_{(0)}$ are polynomials in $\Lambda$ and $\Lambda^{-1}$, respectively, of the form

$$
\begin{aligned}
& B_{(0)}=\Lambda^{N}+b_{1} \Lambda^{N-1}+\cdots+b_{N}, \\
& C_{(0)}=1+c_{1} \Lambda^{-1}+\cdots+c_{N} \Lambda^{-N},
\end{aligned}
$$

where $b_{1}, \ldots, b_{N}$ and $c_{1}, \ldots, c_{N}$ are diagonal matrices. These matrices correspond to finite order difference operators of the form (2). As we show in the next section, the partially factorized forms (39) and (41) - equivalently (11) as difference operators - of $L$ and $\bar{L}^{-1}$ yield a reduction of the Toda hierarchy to the generalized Ablowitz-Ladik hierarchy. In this sense, (40) are initial conditions for a special solution of the generalized Ablowitz-Ladik hierarchy. 
Remark 4. The inverse matrices $B_{(0)}^{-1}$ and $C_{(0)}^{-1}$ are understood to be power series of $\Lambda$ and $\Lambda^{-1}$, respectively:

$$
\begin{aligned}
& B_{(0)}^{-1}=b_{N}^{-1}-b_{N}^{-1} b_{N-1} \Lambda b_{N}^{-1}+\cdots, \\
& C_{(0)}^{-1}=1-c_{1} \Lambda^{-1}+\cdots .
\end{aligned}
$$

Since $B_{(0)}^{-1}$ and $C_{(0)}^{-1}$ are thus interpreted, the inverse of the right hand side of (41) is NOT equal to $D^{-1} B_{(0)} \Lambda^{1-N} C_{(0)}^{-1}=D^{-1} L_{(0)}$. Thus (39) and (41) do not imply the trivial situation where $\bar{L}_{(0)}$ is proportional to $L_{(0)}$.

\section{Generalized Ablowitz-Ladik hierarchy}

The generalized Ablowitz-Ladik hierarchy is defined by the modified Lax equations

$$
\begin{array}{ll}
\frac{\partial B}{\partial t_{k}}=P_{k} B-B Q_{k}, & \frac{\partial B}{\partial \bar{t}_{k}}=\bar{P}_{k} B-B \bar{Q}_{k} \\
\frac{\partial C}{\partial t_{k}}=P_{k} C-C R_{k}, & \frac{\partial C}{\partial \bar{t}_{k}}=\bar{P}_{k} C-C \bar{R}_{k}
\end{array}
$$

for general matrices $B, C$ of the form

$$
\begin{aligned}
& B=\Lambda^{N}+b_{1} \Lambda^{N-1}+\cdots+b_{N}, \\
& C=1+c_{1} \Lambda^{-1}+\cdots+c_{N} \Lambda^{-N}
\end{aligned}
$$

where $b_{1}, \ldots, b_{N}$ and $c_{1}, \ldots, c_{N}$ are diagonal matrices. $P_{k}, Q_{k}, R_{k}$ and $\bar{P}_{k}, \bar{Q}_{k}, \bar{R}_{k}$ are defined as

$$
\begin{array}{ll}
P_{k}=\left(\left(B \Lambda^{1-N} C^{-1}\right)^{k}\right)_{\geq 0}, & \bar{P}_{k}=\left(\left(D C \Lambda^{N-1} B^{-1}\right)^{k}\right)_{<0}, \\
Q_{k}=\left(\left(\Lambda^{1-N} C^{-1} B\right)^{k}\right)_{\geq 0}, & \bar{Q}_{k}=\left(\left(D B^{-1} C \Lambda^{N-1}\right)^{k}\right)_{<0}, \\
R_{k}=\left(\left(C^{-1} B \Lambda^{1-N}\right)^{k}\right)_{\geq 0}, & \bar{R}_{k}=\left(\left(D \Lambda^{N-1} B^{-1} C\right)^{k}\right)_{<0},
\end{array}
$$

and $D$ is an arbitrary constant. The case of $N=1$ amounts to the ordinary Ablowitz-Ladik hierarchy [15].

Remark 5. $D$ may be absorbed into rescaling of the time variables $\bar{t}_{k} \rightarrow$ $D^{-k} \bar{t}_{k}$.

Remark 6. $Q_{k}, R_{k}, \bar{Q}_{k}, \bar{R}_{k}$ satisfy the algebraic relations

$$
Q_{k} \Lambda^{1-N}-\Lambda^{1-N} R_{k}=0, \quad \Lambda^{N-1} \bar{Q}_{k}-\bar{R}_{k} \Lambda^{N-1}=0 .
$$


These relations may be thought of as part of the following "cyclic" reformulation of (43):

$$
\begin{aligned}
\frac{\partial B}{\partial t_{k}} & =P_{k} B-B Q_{k}, & \frac{\partial C}{\partial \bar{t}_{k}} & =\bar{P}_{k} C-C \bar{R}_{k}, \\
\frac{\partial \Lambda^{1-N}}{\partial t_{k}} & =Q_{k} \Lambda^{1-N}-\Lambda^{1-N} R_{k}, & \frac{\partial \Lambda^{N-1}}{\partial \bar{t}_{k}} & =\bar{R}_{k} \Lambda^{N-1}-\Lambda^{N-1} \bar{Q}_{k}, \\
\frac{\partial C^{-1}}{\partial t_{k}} & =R_{k} C^{-1}-C^{-1} P_{k}, & \frac{\partial B^{-1}}{\partial \bar{t}_{k}} & =\bar{Q}_{k} B^{-1}-B^{-1} \bar{P}_{k} .
\end{aligned}
$$

Our goal is to verify that (43) is a consistent reduction of the Toda hierarchy. To avoid unnecessary complications of notations, we consider the case where $D=1$. As remarked above, the general case can be reduced to this case by rescaling $\bar{t}_{k}$ 's.

Let us first confirm that the modified Lax equations (43) imply the Lax equations (24) of the Toda hierarchy for matrices $L$ and $\bar{L}^{-1}$ defined as (39) and (41) suggest.

Lemma 2. If $B$ and $C$ satisfy (43), then

$$
L=B \Lambda^{1-N} C^{-1}, \quad \bar{L}^{-1}=C \Lambda^{N-1} B^{-1}
$$

satisfy the Lax equations (24) of the Toda hierarchy.

Proof. By straightforward calculations, one can derive from (43) and (45) or, equivalently, from (46) - the Lax equations

$$
\begin{aligned}
\frac{\partial}{\partial t_{k}}\left(B \Lambda^{1-N} C^{-1}\right) & =\left[P_{k}, B \Lambda^{1-N} C^{-1}\right] \\
\frac{\partial}{\partial \bar{t}_{k}}\left(B \Lambda^{1-N} C^{-1}\right) & =\left[\bar{P}_{k}, B \Lambda^{1-N} C^{-1}\right] \\
\frac{\partial}{\partial t_{k}}\left(C \Lambda^{N-1} B^{-1}\right) & =\left[P_{k}, C \Lambda^{N-1} B^{-1}\right] \\
\frac{\partial}{\partial \bar{t}_{k}}\left(C \Lambda^{N-1} B^{-1}\right) & =\left[\bar{P}_{k}, C \Lambda^{N-1} B^{-1}\right] .
\end{aligned}
$$

Since

$$
P_{k}=\left(L^{k}\right)_{\geq 0}, \quad \bar{P}_{k}=\left(\bar{L}^{-k}\right)_{<0},
$$

these equations coincide with the Lax equations of the Toda hierarchy.

We can further prove that (43) can be converted to a system of evolution equations for the diagonal matrices $b_{n}, c_{n}$ or, equivalently, the functions $b_{n}(s), c_{n}(s)$ in (2). 
Lemma 3. (43) can be reduced to a system of evolution equations for $b_{n}, c_{n}$, $n=1, \ldots, N$.

Proof. The modified Lax equations (43) have the equivalent (dual) form

$$
\begin{array}{ll}
\frac{\partial B}{\partial t_{k}}=-P_{k}^{c} B+B Q_{k}^{c}, & \frac{\partial B}{\partial \bar{t}_{k}}=-\bar{P}_{k}^{c} B+B \bar{Q}_{k}^{c}, \\
\frac{\partial C}{\partial t_{k}}=-P_{k}^{c} C+C R_{k}^{c}, & \frac{\partial C}{\partial \bar{t}_{k}}=-\bar{P}_{k}^{c} C+C \bar{R}_{k}^{c}
\end{array}
$$

where

$$
\begin{array}{ll}
P_{k}^{c}=\left(\left(B \Lambda^{1-N} C^{-1}\right)^{k}\right)_{<0}, & \left.\bar{P}_{k}^{c}=\left(\left(C \Lambda^{N-1} B^{-1}\right)^{k}\right)\right)_{\geq 0}, \\
Q_{k}^{c}=\left(\left(\Lambda^{1-N} C^{-1} B\right)^{k}\right)_{<0}, & \bar{Q}_{k}^{c}=\left(\left(B^{-1} C \Lambda^{N-1}\right)^{k}\right)_{\geq 0}, \\
R_{k}^{c}=\left(\left(C^{-1} B \Lambda^{1-N}\right)^{k}\right)_{<0}, & \bar{R}_{k}^{c}=\left(\left(\Lambda^{N-1} B^{-1} C\right)^{k}\right)_{\geq 0} .
\end{array}
$$

These two expressions show that each equation of (43) has such a form as

$$
\begin{array}{ll}
\frac{\partial B}{\partial t_{k}}=f_{1, k} \Lambda^{N-1}+\cdots+f_{N, k}, & \frac{\partial B}{\partial \bar{t}_{k}}=\bar{f}_{1, k} \Lambda^{N-1}+\cdots+\bar{f}_{N, k}, \\
\frac{\partial C}{\partial t_{k}}=g_{1, k} \Lambda^{-1}+\cdots+g_{N, k} \Lambda^{-N}, & \frac{\partial C}{\partial \bar{t}_{k}}=\bar{g}_{1, k} \Lambda^{-1}+\cdots+\bar{g}_{N, k} \Lambda^{-N}
\end{array}
$$

hence can be reduced to the evolution equations

$$
\frac{\partial b_{n}}{\partial t_{k}}=f_{n, k}, \quad \frac{\partial c_{n}}{\partial t_{k}}=g_{n, k}, \quad \frac{\partial b_{n}}{\partial \bar{t}_{k}}=\bar{f}_{n, k}, \quad \frac{\partial c_{n}}{\partial \bar{t}_{k}}=\bar{g}_{n, k}
$$

for $b_{n}$ 's and $c_{n}$ 's.

These two lemmas appear to be enough to conclude that (43) defines a reduction of the Toda hierarchy. This is, however, a hasty judgement. We have to confirm the consistency of (43), namely, the commutativity

$$
\begin{aligned}
\frac{\partial}{\partial t_{k}} \frac{\partial B}{\partial t_{l}}=\frac{\partial}{\partial t_{l}} \frac{\partial B}{\partial t_{k}}, & \frac{\partial}{\partial t_{k}} \frac{\partial B}{\partial \bar{t}_{l}}=\frac{\partial}{\partial \bar{t}_{l}} \frac{\partial B}{\partial t_{k}},
\end{aligned}
$$

of flows; otherwise, (43) is not ensured to have non-trivial solutions. This issue can be reduced to deriving a set of zero-curvature equations as shown below. 
Lemma 4. The three sets

$$
\begin{gathered}
\frac{\partial P_{l}}{\partial t_{k}}-\frac{\partial P_{k}}{\partial t_{l}}+\left[P_{l}, P_{k}\right]=0, \quad \frac{\partial \bar{P}_{l}}{\partial \bar{t}_{k}}-\frac{\partial \bar{P}_{k}}{\partial \bar{t}_{l}}+\left[\bar{P}_{l}, \bar{P}_{k}\right]=0, \\
\frac{\partial \bar{P}_{l}}{\partial t_{k}}-\frac{\partial P_{k}}{\partial \bar{t}_{l}}+\left[\bar{P}_{l}, P_{k}\right]=0 \\
\frac{\partial Q_{l}}{\partial t_{k}}-\frac{\partial Q_{k}}{\partial t_{l}}+\left[Q_{l}, Q_{k}\right]=0, \quad \frac{\partial \bar{Q}_{l}}{\partial \bar{t}_{k}}-\frac{\partial \bar{Q}_{k}}{\partial \bar{t}_{l}}+\left[\bar{Q}_{l}, \bar{Q}_{k}\right]=0, \\
\frac{\partial \bar{Q}_{l}}{\partial t_{k}}-\frac{\partial Q_{k}}{\partial \bar{t}_{l}}+\left[\bar{Q}_{l}, Q_{k}\right]=0 \\
\frac{\partial R_{l}}{\partial t_{k}}-\frac{\partial R_{k}}{\partial t_{l}}+\left[R_{l}, R_{k}\right]=0, \quad \frac{\partial \bar{R}_{l}}{\partial \bar{t}_{k}}-\frac{\partial \bar{R}_{k}}{\partial \bar{t}_{l}}+\left[\bar{R}_{l}, \bar{R}_{k}\right]=0 \\
\frac{\partial \bar{R}_{l}}{\partial t_{k}}-\frac{\partial R_{k}}{\partial \bar{t}_{l}}+\left[\bar{R}_{l}, R_{k}\right]=0
\end{gathered}
$$

of zero-curvature equations imply the commutativity (48) of flows.

Proof. The difference of both hand sides of the first equation in (48) can be rewritten as

$$
\begin{aligned}
\text { LHS }- \text { RHS }= & \frac{\partial}{\partial t_{k}}\left(P_{l} B-B Q_{l}\right)-\frac{\partial}{\partial t_{l}}\left(P_{k} B-B Q_{k}\right) \\
= & \frac{\partial P_{l}}{\partial t_{k}} B+P_{l}\left(P_{k} B-B Q_{k}\right)-\left(P_{k} B-B Q_{k}\right) Q_{l}-B \frac{\partial Q_{l}}{\partial t_{k}} \\
& -\frac{\partial P_{k}}{\partial t_{k}} B-P_{k}\left(P_{l} B-B Q_{l}\right)+\left(P_{l} B-B Q_{l}\right) Q_{k}+B \frac{\partial Q_{k}}{\partial t_{l}} \\
= & \left(\frac{\partial P_{l}}{\partial t_{k}}-\frac{\partial P_{k}}{\partial t_{l}}+\left[P_{l}, P_{k}\right]\right) B-B\left(\frac{\partial Q_{l}}{\partial t_{k}}-\frac{\partial Q_{k}}{\partial t_{l}}+\left[Q_{l}, Q_{k}\right]\right) .
\end{aligned}
$$

This vanishes if the first equations of (49) and (50) are satisfied. The other equations of (48) can be derived in the same way.

The zero-curvature equations (49)-(51) are indeed satisfied as we prove below.

Lemma 5. (43) implies the zero-curvature equations (49)-(51).

Proof. According to Lemma 2 ,

$$
L=B \Lambda^{1-N} C^{-1}, \quad \bar{L}^{-1}=C \Lambda^{N-1} B^{-1}
$$


satisfy the Lax equations of the Toda hierarchy. On the other hand, it is well known in the theory of the Toda hierarchy [13] that the Lax equations imply the zero-curvature equations

$$
\begin{gathered}
\frac{\partial\left(L^{l}\right)_{\geq 0}}{\partial t_{k}}-\frac{\partial\left(L^{k}\right)_{\geq 0}}{\partial t_{l}}+\left[\left(L^{l}\right)_{\geq 0},\left(L^{k}\right)_{\geq 0}\right]=0, \\
\frac{\partial\left(\bar{L}^{-l}\right)_{<0}}{\partial t_{k}}-\frac{\partial\left(\bar{L}^{-k}\right)_{<0}}{\partial t_{l}}+\left[\left(\bar{L}^{-l}\right)_{<0},\left(\bar{L}^{-k}\right)_{<0}\right]=0, \\
\frac{\partial\left(\bar{L}^{-l}\right)_{<0}}{\partial t_{k}}-\frac{\partial\left(L^{k}\right)_{\geq 0}}{\partial \bar{t}_{l}}+\left[\left(\bar{L}^{-l}\right)_{<0},\left(L^{k}\right)_{\geq 0}\right]=0 .
\end{gathered}
$$

Since $\left(L^{k}\right)_{\geq 0}$ and $\left(\bar{L}^{-k}\right)_{<0}$ for $L$ and $\bar{L}$ of the foregoing form coincide with $P_{k}$ and $\bar{P}_{k}$, these zero-curvature equations are nothing but (49). In the same sense, both

$$
L=\Lambda^{1-N} C^{-1} B, \quad \bar{L}^{-1}=B^{-1} C \Lambda^{N-1}
$$

and

$$
L=C^{-1} B \Lambda^{1-N}, \quad \bar{L}^{-1}=\Lambda^{N-1} B^{-1} C
$$

satisfy the Lax equations of the Toda hierarchy, and the associated zerocurvature equations are exactly (50) and (51).

We have thus confirmed the following fact. Note that the constant $D$ is restored in the statement.

Proposition 3. (43) defines a consistent system (generalized Ablowitz-Ladik hierarchy) of evolution equations for $b_{n}, c_{n}, n=1, \ldots, N$. This system can be identified with a subsystem of the Toda hierarchy for which the Lax operators $L, \bar{L}^{-1}$ have the factorized form

$$
L=B \Lambda^{1-N} C^{-1}, \quad \bar{L}^{-1}=D C \Lambda^{N-1} B^{-1} .
$$

This result ensures the existence of a solution of (43) with the initial values (40) at $\boldsymbol{t}=\overline{\boldsymbol{t}}=\mathbf{0}$. The associated Lax operators of the Toda hierarchy have the initial values (37)-(38) at $\boldsymbol{t}=\overline{\boldsymbol{t}}=\mathbf{0}$. This leads to the following conclusion.

Proposition 4. The generalized Ablowitz-Ladik hierarchy (43) has a solution with the initial values (40) at $\boldsymbol{t}=\overline{\boldsymbol{t}}=\mathbf{0}$. This solution corresponds to the solution of the Toda hierarchy obtained from (16) by the factorization problem (22). 


\section{Conclusion}

We have thus proved that the generalized Ablowitz-Ladik hierarchy (43) underlies open string amplitudes of topological string theory on the generalized conifolds. These amplitudes are assembled to the tau function $\tau(s, \boldsymbol{t}, \overline{\boldsymbol{t}})$ that is primarily formulated in the fermionic language. To identify it as a solution of the generalized Ablowitz-Ladik hierarchy, we use the dictionary that translates the fermionic setting to the language of $\mathbb{Z} \times \mathbb{Z}$ matrices. The generating operator $g$ of the tau function is thereby converted to the matrix $U$ along with the building blocks such as the fermion bilinears $L_{0}, W_{0}$ and the vertex operators $\Gamma_{ \pm}(z), \Gamma_{ \pm}^{\prime}(z)$. The subsequent consideration is based on these matrices, and can be summarized as follows.

Theorem 1. The matrix $U$ determines a solution of the Toda hierarchy via the factorization problem (22). The associated Lax operators $L, \bar{L}^{-1}$ have the factorized form (53). The factors $B, C$ in (53) are polynomials of the form (44) in the shift matrices $\Lambda, \Lambda^{-1}$ respectively, and satisfy the modified Lax equations (43). Their initial values at $\boldsymbol{t}=\overline{\boldsymbol{t}}=\mathbf{0}$ and the constant $D$ are determined by the Kähler parameters as shown in (40) and (42).

A technical clue of the proof is the fact that the initial values (28) of the Lax operators at $\boldsymbol{t}=\overline{\boldsymbol{t}}=\mathbf{0}$ can be calculated in a very explicit form as shown in (29) -(38). It is ultimately the quantum torus algebra (21) and the matrix-valued quantum dilogarithm (14) that enable us these calculations.

Let us conclude this paper with several remarks.

1. The calculation of initial values can be extended to the Orlov-Schulman operators

$$
M=W \Delta W^{-1}+\sum_{k=1}^{\infty} k t_{k} L^{k}, \quad \bar{M}=\bar{W} \Delta \bar{W}^{-1}-\sum_{k=1}^{\infty} k \bar{t}_{k} \bar{L}^{-k} .
$$

In the context of the quantum torus algebra, it is more natural to consider the exponentials $q^{M}, q^{-\bar{M}}$ rather than $M, \bar{M}$ themselves. Their initial values $q^{M_{(0)}}, q^{-\bar{M}_{(0)}}$ are determined by the initial values (26) $-(27)$ of the dressing operators as

$$
q^{M_{(0)}}=W_{(0)} q^{\Delta} W_{(0)}^{-1}, \quad q^{-\bar{M}_{(0)}}=\bar{W}_{(0)} q^{-\Delta} \bar{W}_{(0)}^{-1} .
$$


One can calculate the right hand side in much the same way as the derivation of (35) and (36):

$$
\begin{aligned}
q^{M_{(0)}} & =\prod_{n=1}^{N}\left(1-Q^{(2 n-1)} q^{\Delta} \Lambda^{-1}\right)\left(1+Q^{(2 n)} q^{\Delta} \Lambda^{-1}\right)^{-1} \cdot q^{\Delta} \\
q^{-\bar{M}_{(0)}} & =\prod_{n=1}^{N}\left(1-Q^{(2 n-1)-1} q^{-\Delta} \Lambda\right)^{-1}\left(1+Q^{(2 n)-1} q^{-\Delta} \Lambda\right) \cdot q^{-\Delta} .
\end{aligned}
$$

These expressions of the initial values may be thought of as yet another realization of "quantum mirror curves" of the generalized conifolds [33, 21]. This issue will be addressed elsewhere.

2. There are some other possible approaches to integrable structures of the type studied in this paper. An approach will be based on unitary matrix models of open string amplitudes [34, 35, 36]. As the ordinary unitary matrix model is linked with the Ablowitz-Ladik hierarchy [6], these matrix models with elliptic weight functions can lead to generalized Ablowitz-Ladik hierarchies. Another way is the line of approach initiated by Brini et al. [8, 15]. In the latest paper [37, they proposed a new scheme of reductions of the Toda hierarchy, which is apparently different from ours, and applied the reduced hierarchy to local Gromov-Witten invariants of a class of orbifolds of the resolved conifold. It is an extremely intriguing problem to compare their results with ours in detail. Since their toric Calabi-Yau threefolds, too, have web diagrams "on a strip", one can construct a Toda tau function from open string amplitudes in exactly the same manner as our case (see Appendix A).

3. It seems extremely difficult to go "beyond the strip". When the web diagram is not on a strip, the open string amplitudes obtained by the topological vertex construction cease to take the simple form $\langle\lambda|g| \mu\rangle$. A way out will be the B-model perspective [38, 39, 40], but the study of integrable structures on the B-model side require quite different techniques. A promising strategy on the A-model side will be the method of "gluing strips" proposed by Eynard et al. [41.

\section{Acknowledgements}

This work is partly supported by JSPS KAKENHI Grant Numbers 24540223 and 25400111. 


\section{A Combinatorial and fermionic expressions of open string amplitudes}

The method of topological vertex [16] provides a combinatorial expression of open string amplitudes for toric Calabi-Yau threefolds. Fundamental building blocks of the construction are the vertex weights 3

$$
C_{\alpha \beta \gamma}=s_{\beta}\left(q^{-\rho}\right) q^{-\kappa(\gamma) / 2} \sum_{\nu \in \mathcal{P}} s_{\alpha / \nu}\left(q^{-{ }^{\mathrm{t}} \beta-\rho}\right) s_{\mathrm{t} \gamma / \nu}\left(q^{-\beta-\rho}\right) .
$$

to be attached to the vertices of the web diagram. These vertex weights are "glued together" along with the weights $\left(-Q_{n}\right)^{\left|\alpha_{n}\right|}$ of the internal edges by summing over the partitions $\alpha_{n}$ assigned to the internal edges. We refer details of the gluing procedure (in which extra "framing factors" $(-1)^{f_{n}} q^{f_{n} \kappa\left(\alpha_{n}\right) / 2}$ are also inserted to adjust the canonical framing of the vertex weights) to Mariño's book [30].

To apply this method to the generalized conifolds, let $\alpha_{0}, \beta_{1}, \cdots, \beta_{2 N}, \alpha_{2 N}$ denote the partitions assigned to the external edges of the web diagram (see Fig. 11). $\alpha_{0}$ and $\alpha_{2 N}$ are on the leftmost and rightmost external edges; $\beta_{1}, \ldots, \beta_{2 N}$ are on the intermediate ones. The amplitude $Z_{\alpha_{0} \beta_{1} \cdots \beta_{2 N} \alpha_{2 N}}$ in this setting becomes the following sum over all partitions $\alpha_{1}, \ldots, \alpha_{2 N}$ on the internal edges:

$$
\begin{aligned}
Z_{\alpha_{0} \beta_{1} \cdots \beta_{2 N} \alpha_{2 N}}= & \sum_{\alpha_{1}, \ldots, \alpha_{2 N} \in \mathcal{P}} C_{\alpha_{1} \beta_{1} \alpha_{0}}\left(-Q_{1}\right)^{\left|\alpha_{1}\right|} C_{\mathrm{t}_{\alpha_{1} \beta_{2} \alpha_{2}}}\left(-Q_{2}\right)^{\left|\alpha_{2}\right|} \\
& \times C_{\alpha_{3} \beta_{3} \alpha_{2}}\left(-Q_{3}\right)^{\left|\alpha_{3}\right|} C_{\mathrm{t}_{\alpha_{3} \beta_{4} \alpha_{4}}\left(-Q_{4}\right)^{\left|\alpha_{4}\right|}} \\
& \times \cdots \\
& \times C_{\alpha_{2 N-1} \beta_{2 N-1} \alpha_{2 N-2}}\left(-Q_{2 N-1}\right)^{\left|\alpha_{2 N-1}\right|} C_{\mathrm{t}_{\alpha_{2 N-1} \beta_{2 N} \alpha_{2 N}}}
\end{aligned}
$$

This combinatorial expression can be translated to the language of fermions as follows. The vertex weights have two equivalent fermionic expressions

$$
\begin{aligned}
& C_{\alpha \beta \gamma}=s_{\beta}\left(q^{-\beta}\right) q^{-\kappa(\gamma) / 2}\left\langle{ }^{\mathrm{t}} \gamma\left|\Gamma_{-}\left(q^{-\beta-\rho}\right) \Gamma_{+}\left(q^{-{ }^{\mathrm{t}} \beta-\rho}\right)\right| \alpha\right\rangle, \\
& C_{\alpha \beta \gamma}=s_{\beta}\left(q^{-\beta}\right) q^{-\kappa(\gamma) / 2}\left\langle{ }^{\mathrm{t}} \alpha\left|\Gamma_{-}^{\prime}\left(q^{-{ }^{\mathrm{t}} \beta-\rho}\right) \Gamma_{+}^{\prime}\left(q^{-\beta-\rho}\right)\right| \gamma\right\rangle
\end{aligned}
$$

as (51) and (66) imply. One can choose either (58) or (59) appropriately in calculations. The edge weights, too, can be expressed as the matrix elements

$$
\left(-Q_{n}\right)^{\left|\alpha_{n}\right|}=\left\langle\alpha_{n}\left|\left(-Q_{n}\right)^{L_{0}}\right| \alpha_{n}\right\rangle
$$

\footnotetext{
${ }^{3}$ The parameter $q$ in the early literature [16] is replaced by $q^{-1}$ in order to fit the crystal model interpretation [11].
} 
of the "diagonal" operator $\left(-Q_{n}\right)^{L_{0}}$ by (3). When these expressions are plugged into (57), the somewhat troublesome factors $q^{\kappa\left(\alpha_{n}\right) / 2}$ on the internal edges turn out to cancel out. (Actually, this is also the case for all web diagrams on a "strip" [17].) Summation over $\alpha_{1}, \ldots, \alpha_{2 N-1} \in \mathcal{P}$ can be interpreted as defining a multiple product of operators in the charge-0 sector of the fermion Fock space. Thus the amplitude becomes, apart from several prefactors, the matrix element of a single operator:

$$
Z_{\alpha_{0} \beta_{1} \ldots \beta_{2 N} \alpha_{2 N}}=s_{\beta_{1}}\left(q^{-\rho}\right) \cdots s_{\beta_{2 N}}\left(q^{-\rho}\right) q^{-\left(\kappa\left(\alpha_{0}\right)+\kappa\left(\alpha_{2 N}\right)\right) / 2}\left\langle{ }^{t} \alpha_{0}\left|g_{\beta_{1} \ldots \beta_{2 N}}\right| \alpha_{2 N}\right\rangle,(60)
$$

where

$$
\begin{aligned}
g_{\beta_{1} \cdots \beta_{2 N}} & \\
= & \Gamma_{-}\left(q^{-\beta_{1}-\rho}\right) \Gamma_{+}\left(q^{-{ }^{\mathrm{t}} \beta_{1}-\rho}\right)\left(-Q_{1}\right)^{L_{0}} \Gamma_{-}^{\prime}\left(q^{-{ }^{\mathrm{t}} \beta_{2}-\rho}\right) \Gamma_{+}^{\prime}\left(q^{-\beta_{2}-\rho}\right)\left(-Q_{2}\right)^{L_{0}} \\
& \times \Gamma_{-}\left(q^{-\beta_{3}-\rho}\right) \Gamma_{+}\left(q^{-{ }^{\mathrm{t}} \beta_{3}-\rho}\right)\left(-Q_{3}\right)^{L_{0}} \Gamma_{-}^{\prime}\left(q^{-{ }^{\mathrm{t}} \beta_{4}-\rho}\right) \Gamma_{+}^{\prime}\left(q^{-\beta_{4}-\rho}\right)\left(-Q_{4}\right)^{L_{0}} \\
& \times \cdots \\
& \times \Gamma_{-}\left(q^{-\beta_{2 N-1}-\rho}\right) \Gamma_{+}\left(q^{-{ }^{\mathrm{t}} \beta_{2 N-1}-\rho}\right)\left(-Q_{2 N-1}\right)^{L_{0}} \Gamma_{-}^{\prime}\left(q^{-{ }^{\mathrm{t}} \beta_{2 N}-\rho}\right) \Gamma_{+}^{\prime}\left(q^{-\beta_{2 N}-\rho}\right) .
\end{aligned}
$$

The operator $g$ of (9) emerges by letting

$$
\alpha_{0}={ }^{\mathrm{t}} \lambda, \quad \beta_{1}=\cdots=\beta_{2 N}=\emptyset, \quad \alpha_{2 N}=\mu
$$

and flipping the sign $Q_{n} \rightarrow-Q_{n}$ of all $Q_{n}$ 's. More precisely, $g$ is related to the operator $g_{\emptyset \ldots \emptyset}$ as

$$
g=\left.q^{W_{0} / 2} g_{\emptyset \cdots \emptyset}\right|_{Q_{n} \rightarrow-Q_{n}} q^{-W_{0} / 2} .
$$

Note here that $q^{ \pm W_{0} / 2}$ are "diagonal" operators with the matrix elements

$$
\left\langle\lambda\left|q^{ \pm W_{0} / 2}\right| \mu\right\rangle=\delta_{\lambda \mu} q^{ \pm(\kappa(\lambda)+|\lambda|) / 2}
$$

as one can see from (3) and (4). Also recall the anti-symmetric property

$$
\kappa\left({ }^{\mathrm{t}} \lambda\right)=-\kappa(\lambda)
$$

of the quadratic Casimir invariant. Consequently, the matrix elements of $g$ in the charge- 0 sector and the open string amplitudes $Z_{\mathrm{t}_{\lambda \emptyset \ldots \emptyset \mu}}$ are related as

$$
\langle\lambda|g| \mu\rangle=\left.q^{(|\lambda|-|\mu|) / 2} Z_{\mathrm{t}} \lambda \emptyset \cdots \emptyset \mu\right|_{Q_{n} \rightarrow-Q_{n}} .
$$

Thus the essential features of the open string amplitudes are fully captured by the matrix elements of $g$. The prefactor $q^{(|\lambda|-|\mu|) / 2}$ is harmless - it can be absorbed into rescaling of the variables $\boldsymbol{x}, \boldsymbol{y}$ of the Schur functions in the generating function (10).

The fermionic expression (60) of open string amplitudes can be generalized to other web diagrams on a strip [18, 19, 20]. It will be obvious that those amplitudes, too, yield a tau function of the Toda hierarchy. 


\section{References}

[1] K. Takasaki, Modified melting crystal model and Ablowitz-Ladik hierarchy J. Phys. A: Math. Theor. 46 (2013), 245202, arXiv:1302.6129 [math-ph].

[2] J. Bryan and R. Pandharipande, The local Gromov-Witten theory of curves, J. Amer. Math. Soc. 21 (2008), 101-136, arXiv:math/0411037.

[3] M. J. Ablowitz and J. F. Ladik, Nonlinear differential-difference equations, J. Math. Phys. 16 (1975), 598-603.

[4] V. E. Vekslerchik, Functional representation of the AblowitzLadik hierarchy, J. Phys. A: Math. Gen. 31 (1998), 1087-1099, arXiv:solv-int/9707008.

[5] S. N. M. Ruijsenaars, Relativistic Toda systems, Comm. Math. Phys. 133 (1990), 217-247.

[6] S. Kharchev, A. Mironov and A. Zhedanov, Faces of relativistic Toda chain, Int. J. Mod. Phys. A12 (1997), 2675-2724, arXiv:hep-th/9606144.

[7] Yu. B. Suris, A note on the integrable discretization of the nonlinear Schrödinger equation, Inverse Problems 13 (1997), 1121-1136, arXiv:solv-int/9701010.

[8] A. Brini, The local Gromov-Witten theory of $\mathbb{C P}^{1}$ and integrable hierarchies, Comm. Math. Phys. 313 (2012), 571-605, arXiv:1002.0582 [math-ph].

[9] T. Nakatsu and K. Takasaki, Melting crystal, quantum torus and Toda hierarchy, Comm. Math. Phys. 285 (2009), 445-468, arXiv:0710.5339 [hep-th].

[10] T. Nakatsu and K. Takasaki, Integrable structure of melting crystal model with external potentials, Adv. Stud. Pure Math. vol. 59 (Mathematical Society of Japan, Tokyo, 2010), pp. 201-223, arXiv:0807.4970 [math-ph].

[11] A. Okounkov, N. Reshetikhin and C. Vafa, Quantum Calabi-Yau and classical crystals, P. Etingof, V. Retakh and I.M. Singer (eds.), The unity of mathematics, Progr. Math. vol. 244 (Birkhäuser, 2006), pp. 597-618, arXiv:hep-th/0309208 
[12] T. Maeda, T. Nakatsu, K. Takasaki and T. Tamakoshi, Five-dimensional supersymmetric Yang-Mills theories and random plane partitions, JHEP 0503 (2005), 056, arXiv:hep-th/0412327.

[13] K. Ueno and K. Takasaki, Toda lattice hierarchy, Adv. Stud. Pure Math. vol. 4 (Kinokuniya, Tokyo, 1984), pp. 1-95.

[14] K. Takasaki and T. Takebe, Integrable hierarchies and dispersionless limit, Rev. Math. Phys. 7 (1995), 743-808, arXiv:hep-th/9405096.

[15] A. Brini, G. Carlet and P. Rossi, Integrable hierarchies and the mirror model of local $\mathbb{C P}^{1}$, Physica D241 (2012), 2156-2167. arXiv:1105.4508 [math.AG].

[16] M. Aganagic, A. Klemm, M. Mariño and C. Vafa, The topological vertex, Comm. Math. Phys. 254 (2005), 425-478, arXiv:hep-th/0305132.

[17] A. Iqbal and A.-K. Kashani-Poor, The vertex on a strip, Adv. Theor. Math. Phys. 10 (2006), 317-343, arXiv:hep-th/0410174.

[18] T. Eguchi and H. Kanno, Geometric transitions, Chern-Simons gauge theory and Veneziano type amplitudes, Phys. Lett. B585 (2004) 163172, arXiv:hep-th/0312223.

[19] K. Nagao, Non-commutative Donaldson-Thomas theory and vertex operators, Geometry and Topology 15 (2011) 1509-1543, arXiv:0910.5477 [math.AG].

[20] P. Sułkowski, Wall-crossing, free fermions and crystal melting, Comm. Math. Phys. 301 (2011), 517-562, arXiv:0910.5485 [hep-th].

[21] K. Takasaki, Remarks on partition functions of topological string theory on generalized conifolds, arXiv:1301.4548 [math-ph].

[22] K. Takasaki, Initial value problem for the Toda lattice hierarchy, Adv. Stud. Pure Math. vol. 4 (Kinokuniya, Tokyo, 1984), pp. 136-163.

[23] T. Nakatsu, K. Takasaki and S. Tsujimaru, Quantum and classical aspects of deformed $c=1$ strings, Nucl. Phys. B443 (1995), 1550-197, arXiv:hep-th/9501038.

[24] K. Takasaki, Toda lattice hierarchy and generalized string equations, Comm. Math. Phys. 181 (1996), 131-156, arXiv:hep-th/9506089. 
[25] T. Miwa, M. Jimbo and E. Date, Solitons: Differential equations, symmetries, and infinite-dimensional algebras, Cambridge University Press, 2000.

[26] A. Okounkov and N. Reshetikhin, Correlation function of Schur process with application to local geometry of a random 3-dimensional Young diagram, J. Amer. Math. Soc. 16, (2003), 581-603, arXiv:math.CO/0107056

[27] J. Bryan and B. Young, Generating functions for coloured 3D Young diagrams and the Donaldson-Thomas invariants of orbifolds, Duke Math. J. 152 (2010), 115-153, arXiv:0802.3948 [math.CO].

[28] I. G. Macdonald, Symmetric functions and Hall polynomials, Oxford University Press, 1995.

[29] T. Takebe, Representation theoretical meanings of the initial value problem for the Toda lattice hierarchy I, Lett. Math. Phys. 21 (1991), 77-84.

[30] M. Mariño, Chern-Simons theory, matrix models, and topological strings, Oxford University Press, 2005.

[31] L. Faddeev and A. Yu. Volkov, Abelian current algebra and the Virasoro algebra on the lattice, Phys. Lett. B315 (1993), 311-318, arXiv:hep-th/9307048.

[32] L. D. Faddeev and R. M. Kashaev, Quantum dilogarithm, Mod. Phys. Lett. A9 (1994), 427-434, arXiv:hep-th/9310070.

[33] S. Gukov and P. Sułkowski, A-polynomial, B-model, and quantization, JHEP 1202 (2012), 070, arXiv:1108.0002v1 [hep-th].

[34] T. Okuda, Derivation of Calabi-Yau crystals from Chern-Simons gauge theory, JHEP 0503 (2005), 047, arXiv:hep-th/0409270.

[35] R. Szabo and M. Tierz, Matrix models and stochastic growth in Donaldson-Thomas theory, J. Math. Phys. 53 (2012), 103502, arXiv:1005.5643 [hep-th].

[36] H. Ooguri, P. Sułkowski and M. Yamazaki, Wall crossing as seen by matrix models, Commun. Math. Phys. 307 (2011), 429-462, arXiv:1005.1293 [hep-th].

[37] A. Brini, G. Carlet, S. Romano and P. Rossi, Rational reductions of the 2D-Toda hierarchy and mirror symmetry, arXiv:1401.5725 [math-ph]. 
[38] M. Aganagic, R. Dijkgraaf, A. Klemm, M. Mariño and C. Vafa, Topological strings and integrable hierarchies, Commun. Math. Phys. 261 (2006), 451-516, arXiv:hep-th/0312085.

[39] R. Dijkgraaf, L. Hollands, P. Sułkowski and C. Vafa, Supersymmetric gauge theories, intersecting branes and free fermions, JHEP 0802 (2008), 106, arXiv:0709.4446 [hep-th].

[40] R. Dijkgraaf, L. Hollands and P. Sułkowski, Quantum curves and DModules, JHEP 0911 (2009), 047, arXiv:0810.4157 [hep-th].

[41] B. Eynard, A.-K. Kashani-Poor and O. Marchal, A matrix model for the topological string I: Deriving the matrix model, arXiv:1003.1737 [hep-th]. 